\title{
Multi Duty Cycle Scheduled Routing in Wireless Sensor Network-lifetime Maximization
}

\author{
Patil Yogita Dattatraya \\ Department of Computer Science and Engineering, Keshav Memorial Institute of Technology, Hyderabad, Telangana, \\ India \\ E-mail: agyogita@gmail.com
}

Jayashree Agarkhed

Department of CSE, Poojya Doddappa Appa College of Engineering, Kalaburagi, Karnataka, India

E-mail: jayashreeptl@yahoo.com

\author{
Siddarama Patil \\ Department of E\&CE, Poojya Doddappa Appa College of Engineering, Kalaburagi, Karnataka, India \\ E-mail: pdapatil@gmail.com
}

Received: 23 October 2020; Accepted: 07 March 2021; Published: 08 October 2021

\begin{abstract}
Cluster-based protocols are best for applications that require reliability and a continuous functioning environment with a sustainable lifetime of WSN. The dynamic nature of the sensor node makes energy conservation a challenging issue. Sensor node scheduled based on sensing error for energy conservation compromise the accuracy of prediction. The high data accuracy achieved using a single duty cycle controller at each node with compromised throughput and increased routing overhead. Duty Cycle Controller managing a great number of control messages at the network level leads to control packet interference with data packet transmission, increasing packet drop and minimizing throughput. Also, the single-duty cycle controller at the network level leads to increased control overhead. The proposed multilevel cluster-based approach focuses on the appropriate cluster design, selection of cluster head, and sensor nodes scheduling based on sensing error. The proposed method applies a multi-duty cycle controller at each cluster level, and control messages handled are related to nodes in a cluster. Thus has less interference and packet drop leading to maximum throughput than existing methods. The simulation results demonstrated that the proposed method with sensor nodes scheduled at individual cluster levels using a multi-duty cycle controller exhibited improved network lifetime, throughput, and reduced energy consumption compared with the state-of-the-art techniques.
\end{abstract}

Index Terms: Clustering, Energy efficiency, Error prediction, Routing, WSN.

\section{Introduction}

The [1 -3] nodes in WSN have limits on power supply due to its difficulty of recharging in the harsh and remote environment. There also exists constraint on prolonged operation due to the limited bandwidth available for communication, processing speed, and memory capacity [4]. These limitations have given rise to many research works which exclusively focused on the maximizing the utilization of limited sensor resources [5,6]. The clustering overcomes the efficient use of power during data transmission. Clustering improves network scalability, balances network traffic, and reduces the routing table size at the individual node [7]. Also, save up communication bandwidth in inter-cluster routing among $\mathrm{CHs}$ [8] and provide stabilized network topology [9].

For energy conservation, $\mathrm{CH}$ schedules activities in the cluster by switching the node in the active or sleep state [10-13]. Another advantage of clustering is that $\mathrm{CH}$ aggregates the data from cluster member nodes in its respective cluster to minimize packet count to be sent [14] [15]. Energy conservation achieved by clustering of sensor nodes sensing activities leads to power consumption. Many applications [16] require sensor nodes to sustain for weeks or evenly months together. Thus there is a need to avoid unnecessary sensing activities to achieve an extended lifetime of all sensor nodes. Scheduling algorithms have developed to turn on the sensor when required and turn off the sensor whenever needed to save energy.

The proposed work, M ulti-Duty cycle Scheduled Routing in Wireless Sensor Network-lifetime maximization (MDCSR), employs dynamic scheduling based on sensing error among collaborative sensors to optimize the tradeoff between energy consumption and the accuracy of predictions. The proposed scheme has an advantage over single node scheduling methods called "eSENSE energy-efficient stochastic sensing framework for wireless sensor platforms" (IES) 
[17]. Sensor nodes are scheduled for data transmission and provide an effective way to conserve energy. It also reduces transmitting and sensing power while preserving sensing quality. Another critical parameter to increase the lifetime of the sensor node is to reduce power consumption. The MDCSR is an extension of Error Prediction Scheduling for Energy Efficient Routing in Wireless Sensor Network [18] with a simulation graph obtained by a varying number of nodes, intervals, and simulation time. The issue of control overhead due to single DCC at the network level in [19] Collaborative Scheduling in Dynamic Environments using Error Inference (CIES) overcome by multiple DCC in MDCSR.

The organization of the remaining part is as follows. Section 2 details related works, Section 3 specifies the methodology, and Section 4 discusses system design and working of MDCSR. In section 5, the performance of MDCSR compared with CIES and IES varying the number of nodes, interval, and simulation time. Section 6 concludes the paper.

\section{Related Work}

The research work carried out on collaborative sensing [19-22] specifies an efficient way to select a minimum number of sensor nodes to offer better coverage. Much of the existing work has focused on sensing activities based on coverage requirements. There is still scope to schedule sensing activities based on sensing error to provide data accuracy within desirable bounds.

Error Predictor model, referred to as the non-collaborative method, performs local error prediction. The noncollaborative system that is eSense uses this method. Sensors do not sense data continuously, and data reconstructed using an empirical model [23, 24], which is best for environmental monitoring. The sensor nodes use a local error predictor to predict the status of the environment and the actual data sensed by the sensor compared with the value indicated by the predictor. The error predictor generates a prediction error e_ifor each node i. If the sensed data is not the same as the predicted value, switch the node to the sleep state. Else node remains in active state.

With a sensing and scheduling algorithm called CIES in [19], nodes share sensing error information and control sensing errors through neighborhood coordination. Also, the network can respond to dramatic environmental changes more quickly and can be used for monitoring applications to provide high data accuracy while conserving energy. The local sensing scheduler [22] uses the error information to schedule a single node in either sleep or active state. CIES share the error information among the neighbor nodes. The neighbor nodes trigger the sensing activity of other nodes if the inferred errors exceed the error tolerance.

In the existing approach, CIES the single duty cycle controller employed among all clusters in the network, which minimizes throughput due to message overhead during the scheduling of sensor nodes. The MDCSR cluster-based approach resolved the issue. The cluster-based approach ensures energy consumption as discussed in [25] by a selection of $\mathrm{CH}$ based on either, node density, residual energy metric, the average energy of the network, node degree, etc.

In [26], the division of the network into an optimal number of sectors, selection of the optimal number of $\mathrm{CH}$, and then initialization of the network with one node as a $\mathrm{CH}$ in each sector and selection of the node with highest residual energy in the cluster as a vice $\mathrm{CH}$ helps to maintain the optimal number of $\mathrm{CH}$ s throughout the network lifetime. The author in [27] proposed an efficient clustering algorithm using spectral analysis, domain knowledge, and split-mergerefine approach to enhance the efficiency, quality and minimizes empty clusters.

The MDCSR employs multi-level clustering with a unique duty cycle controller (DCC) to schedule the nodes on sensing error in each cluster.

\section{Methodology}

Appropriate design of cluster and selection of $\mathrm{CH}$ minimize energy consumption during message communication and aggregation, which is one of the biggest design issues. The immense application of WSN in every field can be expanded by the efficient design of routing with its limited storage capability and battery life. The proposed method uses multi-level clustering to minimize energy consumption. Since member nodes communicate with the sub-cluster head and sub-cluster head communicate with the primary cluster head at next level in multi-level clustering, the transmission power required is less, and also interference is subjected to nodes within the cluster. Multi-level clusterbased architecture helps to minimize delay due to constant path from nodes within the cluster to $\mathrm{CH}$ and from $\mathrm{CH}$ to primary $\mathrm{CH}$ at the next level to sink even with the increase in the number of nodes. Thus multi-level clustering achieves scalability, energy conservation, minimizes routing overhead and interface. For optimal energy balance routing, there is a need to develop efficient sensing and scheduling algorithm. Single DCC in CIES works at the network level. Each node performs neighbor inferred error estimation and shares the information for scheduling among many nodes in a network, which incurs more delay due to control message transmission. While in MDCSR, DCC works at the cluster level. DCC at the individual cluster carries out node error estimation and scheduling. Compared to non-collaborative approaches, the proposed approach meets performance requirements, i.e., increased network lifetime and throughput. 


\section{System Design and Working of MDCSR}

The findings of the literature survey motivate to accomplish the following goals.

1. To develop a scheduling algorithm using a multi-duty cycle controller to increase network lifetime.

2. To achieve high throughput with minimized routing overhead using multilevel clustering.

These goals are realized by cluster-based architecture, as discussed next.

\subsection{Cluster-based architecture}

As depicted in figure 1, primary $\mathrm{CH}(\mathrm{PCH})$ selected as a node with the highest residual energy for every round of time period $\mathrm{T}$ along with sub-cluster formation. The $\mathrm{PCH}$ formed at level 3 in multilevel clustering. In respective clusters, a node with the highest residual energy was selected as $\mathrm{CH}$ at level 2. Another node with the highest energy among the remaining nodes in the respective cluster was chosen as DCC. In each chosen sub-cluster, the node is selected in the range 'R'. All member nodes are at level 1. Each DCC uses the Error prediction system to schedule nodes in the sub-cluster for energy balancing among cluster member (CM) nodes. The following section discusses the working of MDCSR.

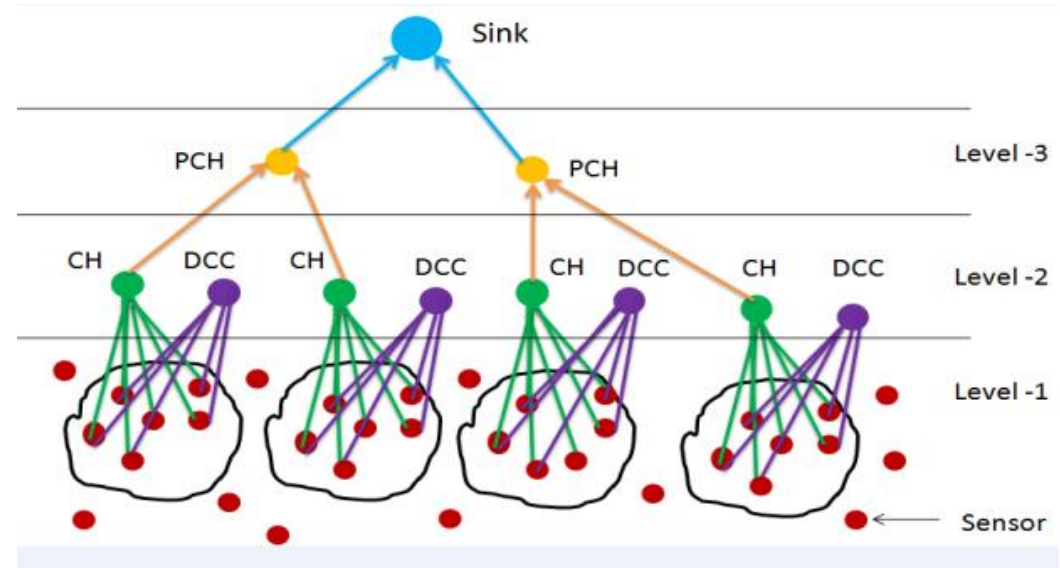

Fig.1. Cluster-based architecture for node energy balanced routing

\subsection{Working of MDCSR}

The MDCSR uses an error predictor shown in figure 2, generates prediction error $\mathrm{e}_{\mathrm{i}}$ for each node $i$.

The sensed data sent as input to the predictor to estimate error. The predicted error sent to DCC performs error prediction at the neighbor node to compute the inferred error.

If the sensed data is not the same as the predicted value, store error, then store error $\mathrm{e}_{\mathrm{i}}$ else considers predicted value. Each DCC in the cluster has information of all the CMs in a cluster. It collects observation $\left\{o b_{1}^{i}, o b_{2}^{i}, \ldots, o b_{n}^{i}\right\} o f$ each node $i$, where $\mathrm{n}$ denotes ' $\mathrm{n}$ ' number of observations of node $\mathrm{i}$. The observation vector obtained at time $T=$ $\left\{t_{1}^{i}, t_{2}^{i}, \ldots, t_{n}^{i}\right\}$. The process is repeated for each round to analyze sensing correlation among the nodes. The observation vector helps to compute the weight value $W(i, j)$ between node $i$ and node $j$ in the next step. Each MDCSR compares the active sensor node sensing value with the predicted value of the error predictor. The prediction error determines observation error $e_{i}^{n}$ at sensor node $i$.

At node $\mathrm{j}$ the weighted average inferred error $W_{e_{j}}$ is calculated as

$$
W_{e_{j}}=\frac{\sum_{k} e_{k j} \times W_{k j}}{W_{k j}}
$$

where,

$k \in N(j)$

$k$ is a neighbor node of the node $j$

$N(j)$ is a list of neighbor nodes of node $\mathrm{j}$

If $W_{e_{j}}$ greater than the threshold value, then the neighbor node with a high weight value will have a greater chance of violating data accuracy. Then such nodes are made to sleep, and node $\mathrm{j}$ is switched to an active state. 


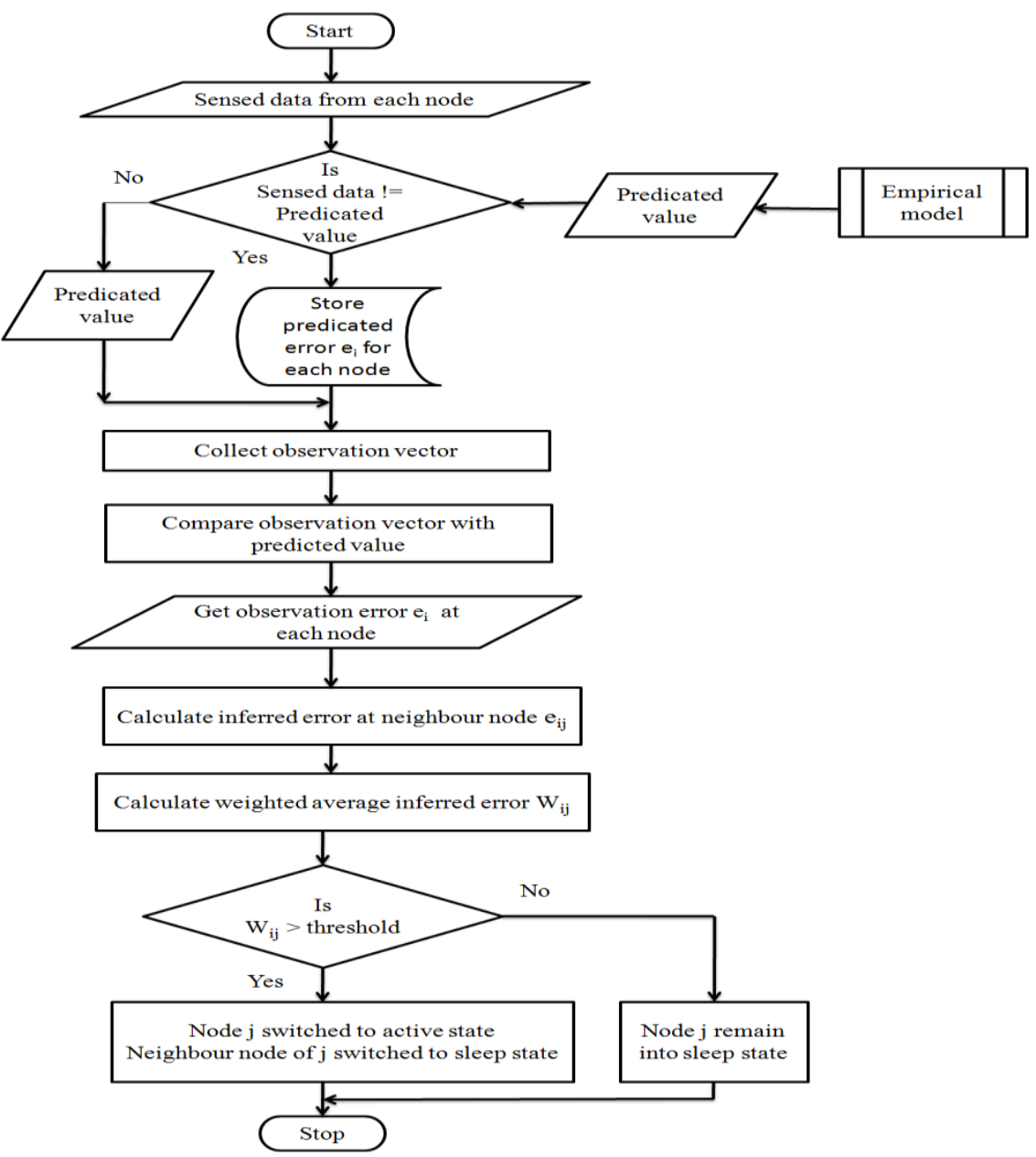

Fig.2. Depicts the working of MDCSR through flowchart representation.

\subsection{Implementation of MDCSR}

The MDCSR incorporates multiple DCC to provide energy-efficient data transmission in clustered-based WSN as discussed in algorithm Lifetime maximization using MDCSR.

\section{Algorithm: Lifetime maximization using MDCSR}

Step 1: Perform node deployment

Step 2: Select Primary $\mathrm{CH}$ as a node with the highest energy in a separate region of the sensor field at level 2.

Step 3: Primary $\mathrm{CH}$ forms clusters of nodes that are within range $\mathrm{R}$.

Step 4: Select sub-cluster $\mathrm{CH}$ as a node with the highest energy among all nodes in each sub-cluster at level 1.

Step 5: Select DCC in each sub-cluster

Step 6: Calculate inferred error at each node

Step 7: Collect an observation vector

Step 8: Get observation error $e_{i}$ at each node by comparison of the observation vector with predicted value

Step 9: Compute at sensor node $\mathrm{i}$, the inferred error $e_{i j}$ at neighbor sensor $\mathrm{j}$ using probability density mass function

Step 10: Calculate weighted average inferred error at neighbor node as per equation 1

Step 11: If $W_{e_{j}}>e_{t}$ then //neighbor node of a node $\mathrm{j}$ violates data accuracy

Step 11.1: Sensor node $\mathrm{j}$ switched to active mode by DCC

Step 11.2: Neighbor node i with high $e_{i j}$ switched to sleep state else

Step 11.1: Node $\mathrm{j}$ is in sleep mode

Step 12: Repeat step 6 to step 11 for every round and go to step 2 at the end of each round. 
The MDCSR provides energy-efficient data transmission in the cluster-based WSN. The sensor nodes are randomly deployed and are assumed to be homogeneous. After deployment, the neighbor node sends the hello packets to ensure their availability. The residual energy of each node was calculated, and a set of PCH was selected with the highest energy for each round of time T. Next, sub-clusters formed by grouping nodes in a specific range. In each subcluster, $\mathrm{CH}$ and multiple DCC are selected. The MDCSR carries out an error prediction method for scheduling nodes. The error predictor predicts inferred error. Next, error prediction estimates and compare the error concerning the neighbor nodes compared with error tolerance. If the estimated error is higher than the error tolerance value, then the MDCSR switches the node $\mathrm{j}$ to active mode, and neighbor node $\mathrm{i}$ of node $\mathrm{j}$ turned to sleep mode. Else node $\mathrm{j}$ remains in sleep mode. For data transmission, each $\mathrm{CM}$ at level 2 transmits sensed data to its respective $\mathrm{CH}$. Each $\mathrm{CH}$ at level 2 sends data to $\mathrm{PCH}$ at level 3. The $\mathrm{PCH}$ sends data to the sink.

\section{Simulation and Analysis of Performance}

The MDCSR performance was evaluated using an event-driven simulate, NS2 simulator [28]. The simulation environment has been discussed in the following subsection.

\subsection{Simulation Environment}

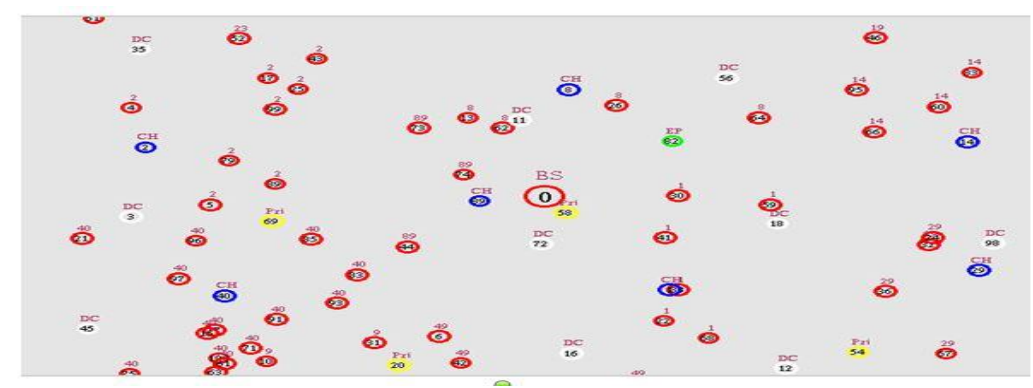

Fig.3. Simulation scenario

The network area of 500 X 500 established using a clustering approach. Figure 3 shows the simulation scenario with cluster-based topology with 100 nodes and one sink node for each node channel capacity set to 3e6. MAC layer protocol uses IEEE 802.11, and the simulated traffic is CBR. The simulation runs for 200 seconds. The performance evaluated varying the number of nodes and changing the number of packets per second.

\subsection{Simulation Parameters}

The simulation conducted using parameters listed in table 1.

Table 1. Simulation parameters

\begin{tabular}{|c|c|}
\hline Parameter & Value \\
\hline Total no. of nodes & 101 \\
\hline Area & $500 \times 500$ \\
\hline Mac & 802.11 \\
\hline Simulation time (seconds) & 200 \\
\hline Traffic source & CBR \\
\hline Transmit Power $(\mathrm{J})$ & 0.2 \\
\hline Receiving Power $(\mathrm{J})$ & 0.1 \\
\hline Initial energy $(\mathrm{J})$ & 100 \\
\hline
\end{tabular}

\subsection{Performance Evaluation Metrics}

The performance of MDCSR has been evaluated using an event-driven simulate, NS2 simulator [29], considering the following QoS metrics [30].

1. Error Rate: Count of errors that result from the difference between sensed value (by the sensor) and predicted value (by predictor) defined as error rate.

$$
\text { Error Rate }=\sum \text { Sensed value }- \text { predicted value }
$$

2. Miss Ratio: The Miss ratio is the ratio of total $W_{e_{j}}$ that are less than $\mathrm{e}_{\mathrm{t}}$ to total event generated. 


$$
\text { Miss ratio }=\frac{\sum W_{e_{j}}<e_{t}}{\text { Number of events }}
$$

where,

$e_{t}=$ Error tolerance or threshold

3. Average Energy Consumption: It is a measure of the total energy consumed by all $\mathrm{N}$ sensor nodes during the data transmission operation.

$$
\text { Average Energy Consumption }=\frac{\sum E_{i}-E_{r}}{N}
$$

where,

$E_{i}:$ Initial energy

$E_{r}$ : remaining energy

$i=1$ to $\mathrm{N}$ nodes

4. Control overhead: The control overhead used to compute routing overhead. Control overhead indicates the count of control messages generated apart from data packets generated.

$$
\text { Control Overhead }=\text { count of control messages generated }
$$

5. Routing overhead: The ratio of control packets generated by the routing protocol to total data packets generated is defined as routing overhead.

$$
\text { Routing Overhead }=\frac{\text { Number of control packets sent }}{\text { Total number of data packets sent }}
$$

\subsection{Results}

Performance of the proposed work MDCSR compared with existing work IES and CIES. The simulation results prove that MDCSR outperforms IES and CIES. In this section, the results obtained:

- By varying the number of nodes

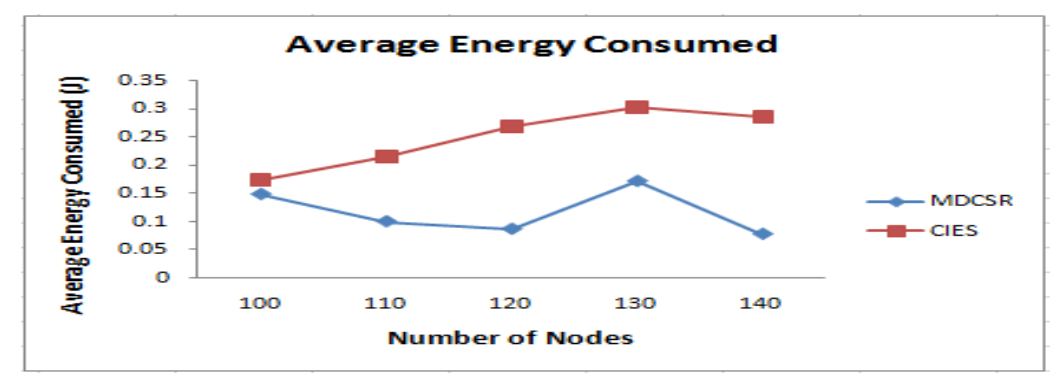

Fig.4. Average Energy Consumed Versus Node

Figure 4 depicts that the average energy consumed by MDCSR is less than CIES, even with an increase in the number of nodes. As the number of nodes increases, there are many nodes switched to active mode resulting in a reduced error rate. With the increase in node density (say 120 nodes onwards), more nodes are active and increase energy consumption. Further, these nodes incurred an increased error rate and switched to a sleep state. Hence lead to less average energy consumption for nodes more than 130. The increase in node density leads to more clusters with respective $\mathrm{CH}$, Duty cycle controller, and $\mathrm{PCH}$. The transmission range minimization between $\mathrm{CM}$ and $\mathrm{CH}$ and routing overhead limited to cluster results in less average energy consumption at 140 nodes. At 140 nodes, next-hop distance from $\mathrm{CM}$ to $\mathrm{CH}$ and from $\mathrm{CH}$ to $\mathrm{PCH}$ decreases which in turn leads to less average energy consumption due to multilevel clustering 


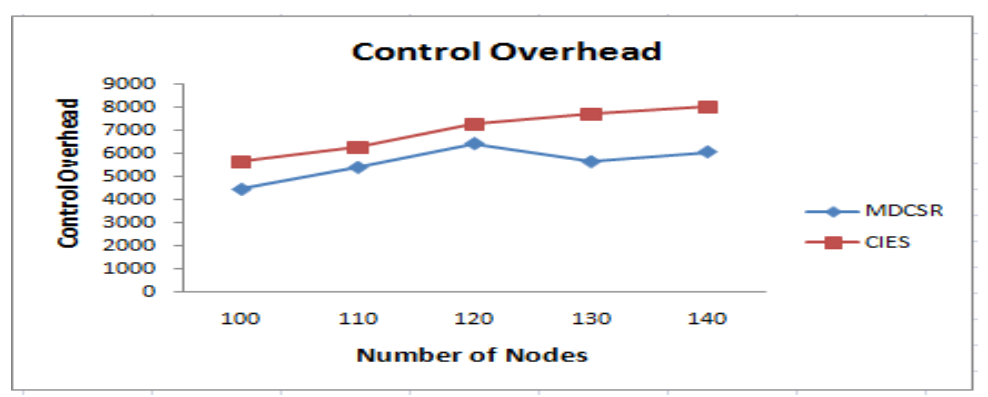

Fig.5. Control Overhead Versus Node

Figure 5 depicts that Control Overhead with CIES is more, as DCC works at the network level while in MDCSR, DCC works at the cluster level. The transmission of an observation vector between the DCC and CM is limited to total nodes in a cluster and has less control overhead.

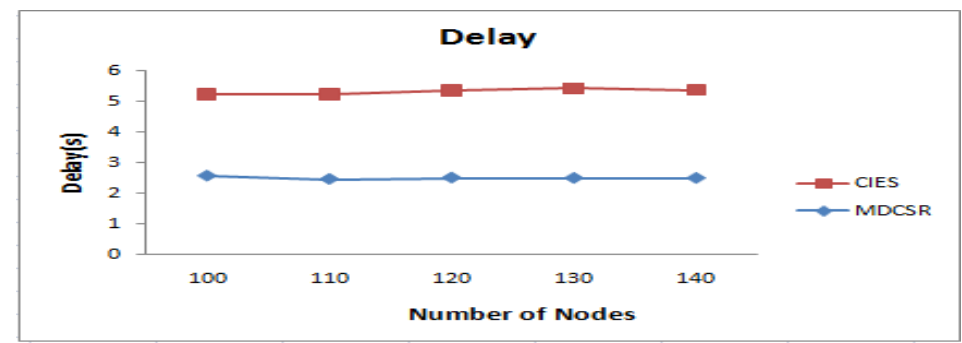

Fig.6. Delay Versus number of nodes

Figure 6 depicts that delay incurred by CIES is $25 \%$ more compared to MDCSR. The DCC in CIES works at the network level. Each node performs neighbour inferred error estimation and shares the information for scheduling among many nodes in a network, which incurs more delay. While in MDCSR, DCC works at the cluster level. DCC at the individual cluster carries out node error estimation and scheduling. The increase in the number of nodes increases the number of clusters while the link connected from the level 1 nodes to level 2 nodes and link connected from the level 2 nodes to level 3 nodes remain the same in multi-level clustered routing. Hence, the delay from each cluster member node to sink remains the same.

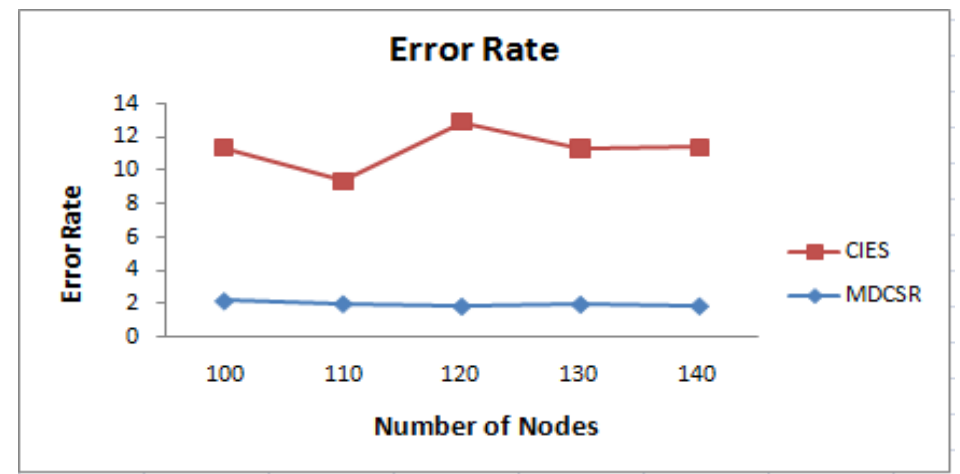

Fig.7. Error Rate Versus the number of nodes

Figure 7 depicts that by using cluster-based architecture in MDCSR, all CMs are scheduled based on error rate. As the node density increases, the number of nodes available to be awakened by the DCC in the respective cluster increases and reduces the error rate. The error rate with MDCSR is $70 \%$ less than CIES.

Figure 8 depicts that jitter induced by MDCSR decreases with an increase in node density as the error rate is less with an increase in node density. More nodes are involved in data transmission without causing the delay. CIES incurs a reduced value of jitter but is comparatively more than MDCSR jitter value by $20 \%$.

Figure 9 depicts that the miss ratio in the case of MDCSR is $45 \%$ less than CIES, with an increase in node density. As nodes are switched to active mode immediately, a high weighted inferred error leads to a less miss ratio.

Figure 10 depicts that overall residual energy is high using MDCSR than CIES, even with an increase in node number. As the number of nodes increases, more nodes switch to active mode, resulting in less node energy consumption. In the case of CIES, as the number of nodes increases, it incurs more message transmission to get the observation vector status among the sensor nodes in the network and reduces node residual energy. 


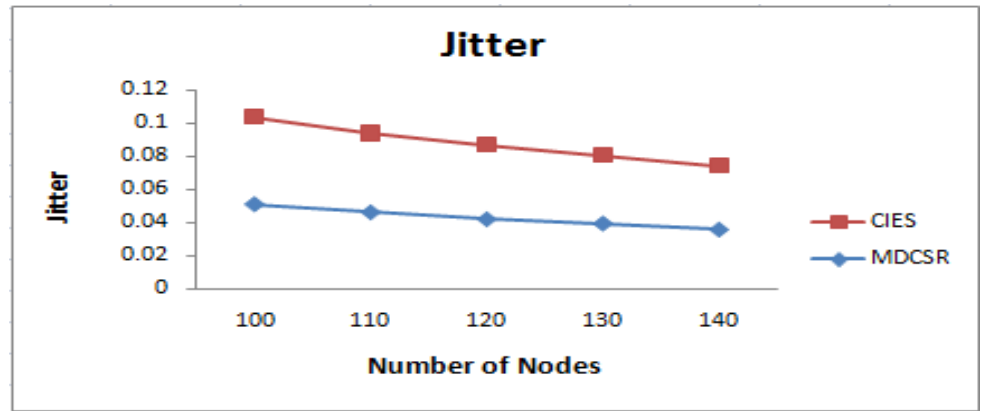

Fig.8. Jitter Versus the number of nodes

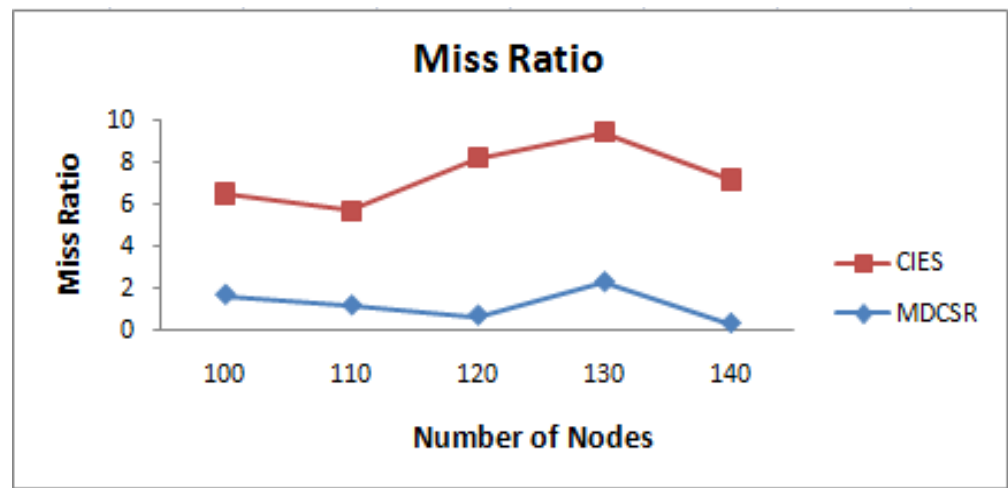

Fig.9. Miss Ratio Versus the number of nodes

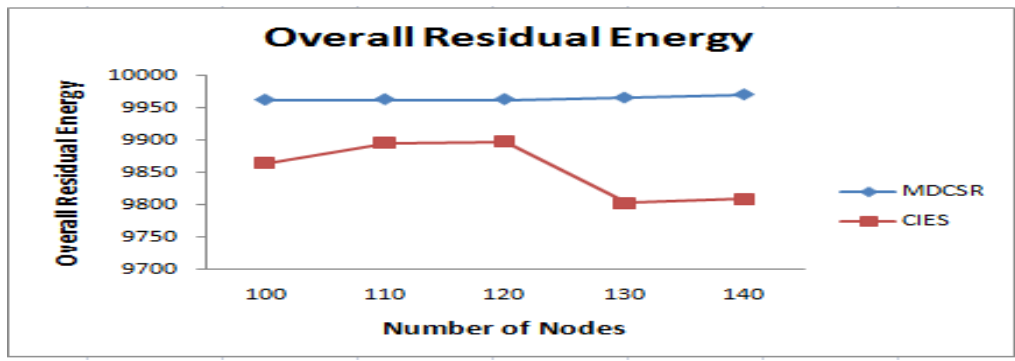

Fig.10. Overall Residual Energy Versus the number of nodes

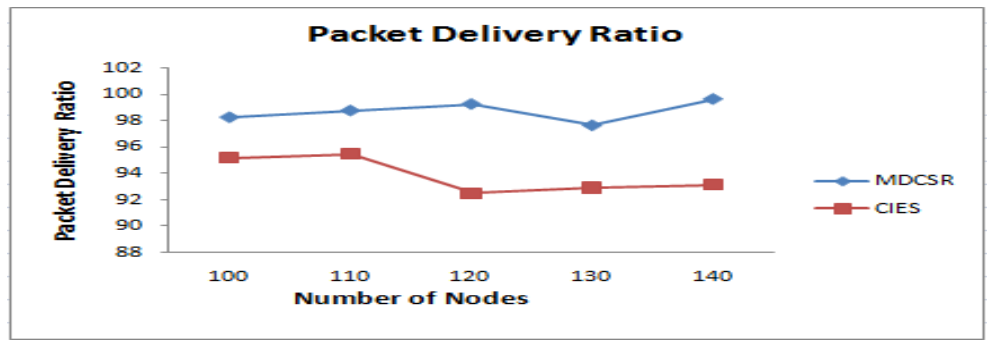

Fig.11. Packet Delivery Ratio Versus the number of nodes

Figure 11 depicts that the packet delivery ratio using MDCSR is more than CIES by using a cluster-based approach and by the scheduling of node at the cluster level.

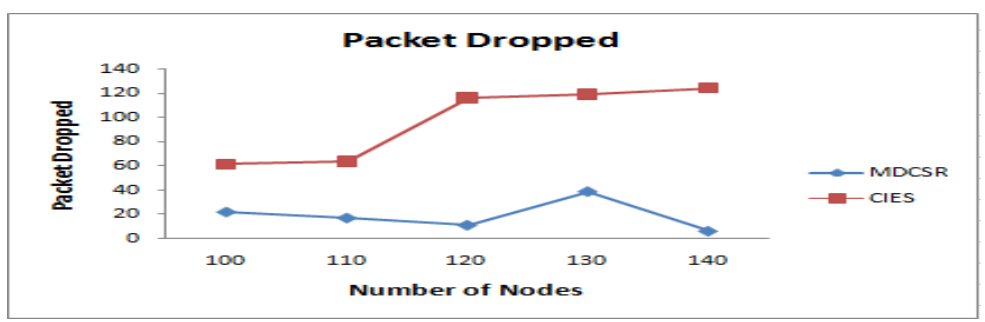

Fig.12. Packet Dropped Versus the number of nodes 
Figure 12 depicts that the number of packets dropped by MDCSR is relatively minimal, with an increase in node count. The increase in node density increases the number of clusters, and intra-cluster communication is handled by respective $\mathrm{CH}$ using the TDMA schedule. Further avoids interference among the CMs in an individual cluster and packet drop.

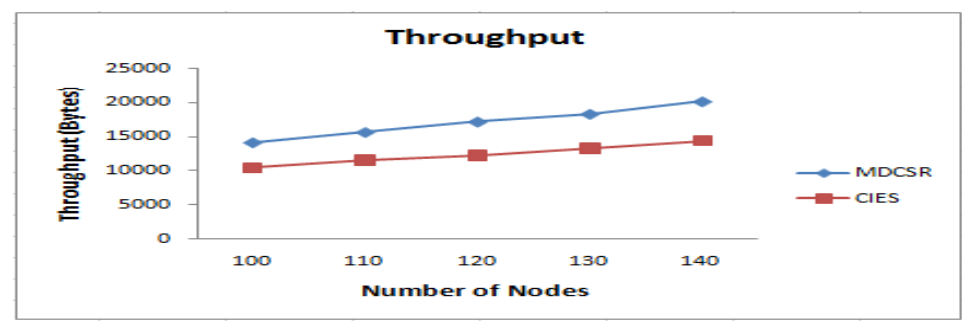

Fig.13. Throughput Versus the number of nodes

Figure 13 depicts that throughput achieved using MDCSR is 50\% more than CIES due to simultaneous transmission from CMs to $\mathrm{CH}$ in the respective cluster. As DCC in CIES manages a large number of control messages at the network level leads to control packet interference with data packet transmission, increasing packet drop and minimizing throughput. In the case of MDCSR, DCC works at the cluster level, and control messages are related to nodes in a cluster. Thus has less interference and packet drop leading to maximum throughput than CIES.

- By varying Interval

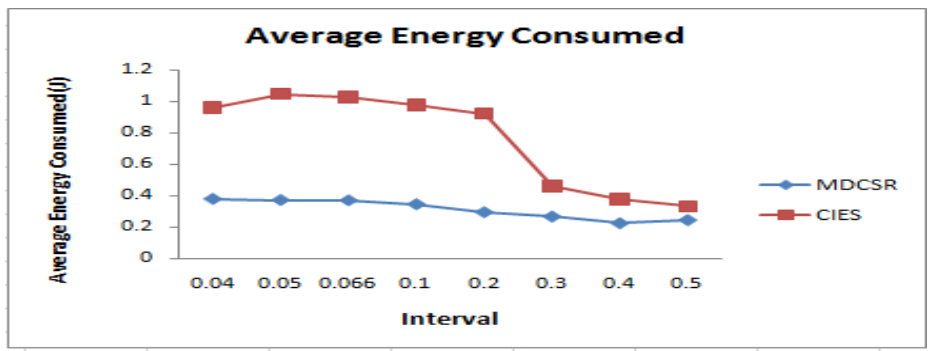

Fig.14. Average Energy consumption Versus Interval

Figure 14 depicts that the average energy consumed using MDCSR is less with an increase in the number of packets per second than CIES. As the number of packets increases in MDCSR, the node's residual energy decreases and increases the error rate. The DCC switches the node with high weighted error to sleep mode, minimizing average energy consumption. In the case of CIES, an increase in the number of packets causes more interference and the dropping of packets. The retransmission due to dropped packets leads to more average energy consumption.

Figure 15 depicts that control overhead increases with an increase in the number of packets per second using MDCSR but is relatively $40 \%$ less than CIES. The control message transmission is less and limited to the number of nodes in a cluster in MDCSR. CIES handles control messages among all the nodes in a network using a single DCC and results in more control overhead.

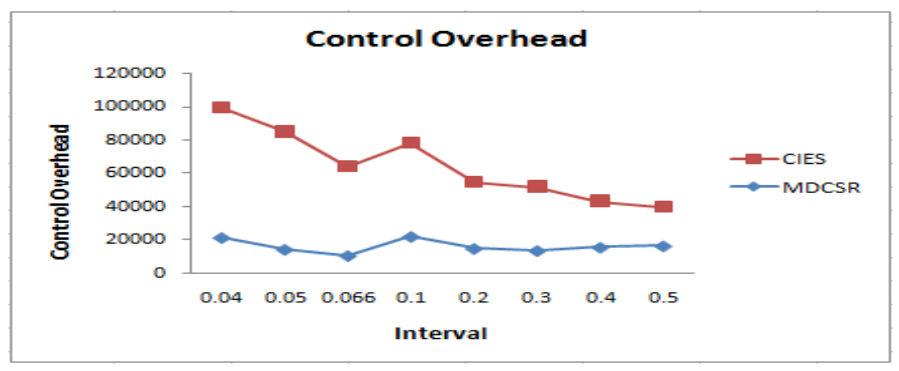

Fig.15. Control Overhead Versus Interval

Figure 16 depicts that the error rate incurred using MDCSR is relatively minimal, with an increase in the number of packets per second compared to CIES. Transmission of data packets at a specific TDMA schedule in MDCSR does not cause packet drop as in CIES by more interference due to significant packet rate. Packet drop leads to the retransmission of lost packet exhaustion node with more energy consumption, leading to more error rate. 


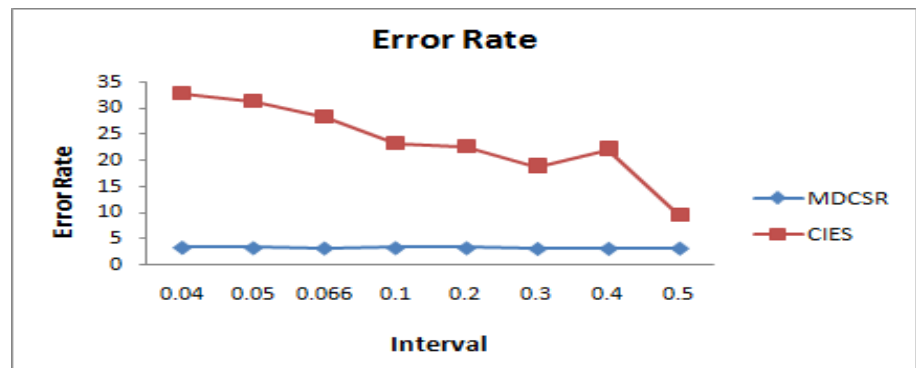

Fig.16. Error Rate Versus Interval

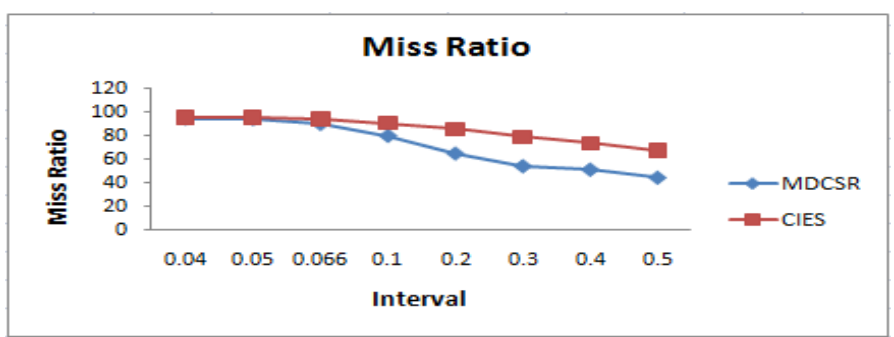

Fig.17. Miss Ratio Versus Interval

Fig. 17 depicts that the miss ratio using MDCSR increases with an increase in the number of packets but is relatively less than CIES. At the five packets per second, MDSCR achieves minimized miss ratio than CIES. Transmission of increased packet rate consumes more energy of nodes on the dedicated path, leading to a high miss ratio.

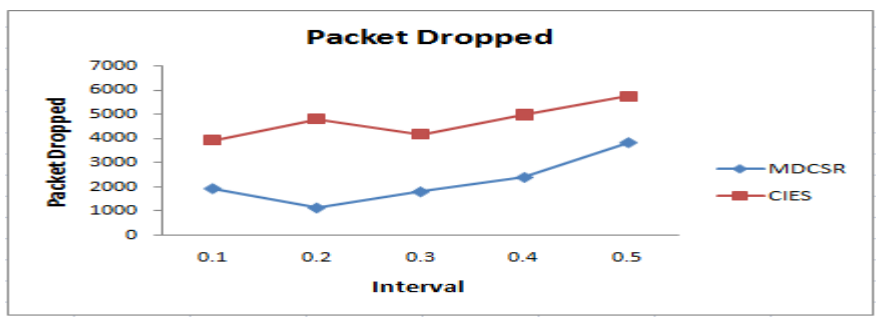

Fig.18. Packet Dropped Versus Interval

Figure 18 depicts that the number of packets dropped using MDCSR is minimal compared to CIES, even with an increase in the number of the data packets as packet transmission from $\mathrm{CM}$ to $\mathrm{CH}$ is performed at the specified schedule using TDMA.

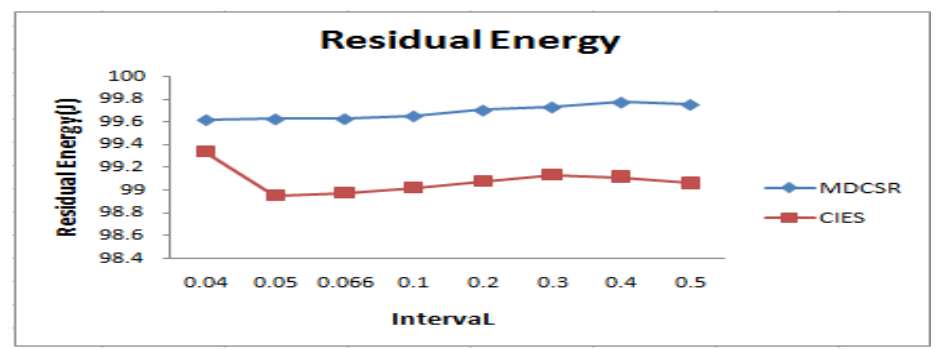

Fig.19. Residual Energy Versus Interval

Figure 19 depicts the low value of residual energy with an increase in the number of packets. To forward an increased number of packets per second, the sensor node energy utilized increases, resulting in a low value of the residual energy of a node. MDCSR incurs less consumption of residual energy compared to CIES.

Figure 20 depicts that throughput achieved using MDCSR is comparatively more than CIES using the clusterbased approach even with the increase in the number of packets per second. In CIES, an increase in the number of packets increase buffering delay, and in turn, leads to packet drop and retransmission. Retransmission of packet increase delay and minimize the throughput in CIES. MDCSR has minimal packet drop due to clustering nodes, achieving high throughput. 


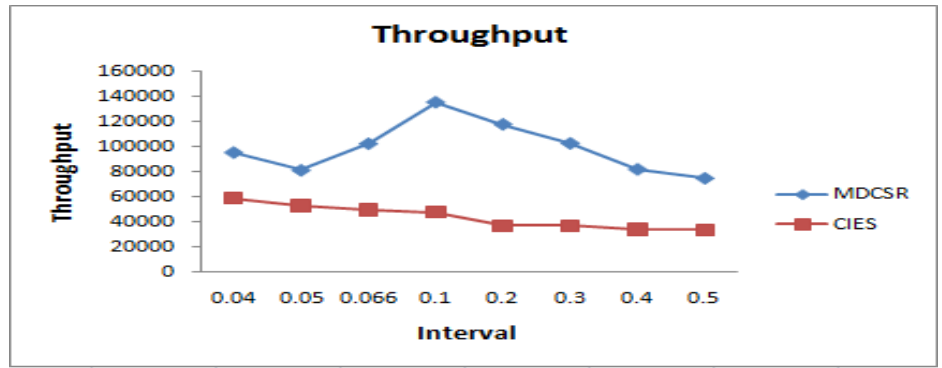

Fig.20. Throughput Versus Interval

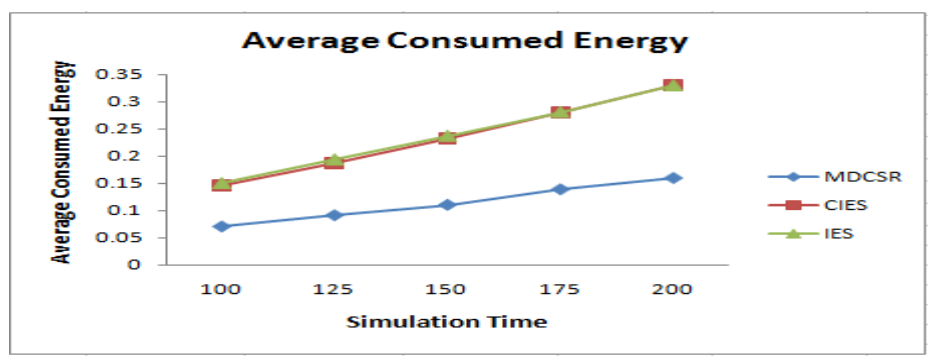

Fig.21. Simulation Time Versus Average Consumed Energy

Figure 21 shows the average consumed energy in the case of MDCSR increases with an increase in simulation time to maintain data accuracy for specific applications. This increase in average consumed energy is relatively less than $20 \%$ compared to CIES and IES. The average consumed energy with IES and CIES is $35 \%$ and with MDCSR is just $15 \%$.

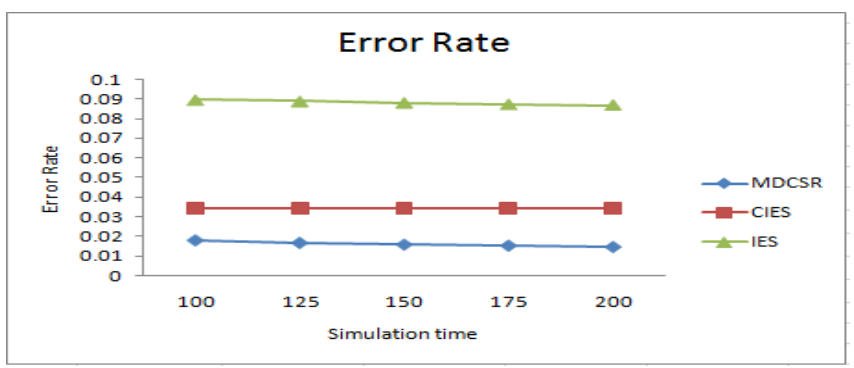

Fig.22. Error Rate Versus Simulation Time

Figure 22 shows the error rate versus simulation time. As IES performs error estimation at the node level, it cannot respond to a drastic change in the environment. These changes quickly lead to more error rates compared to CIES. CIES has error rates of $20 \%$ more than MDCSR.

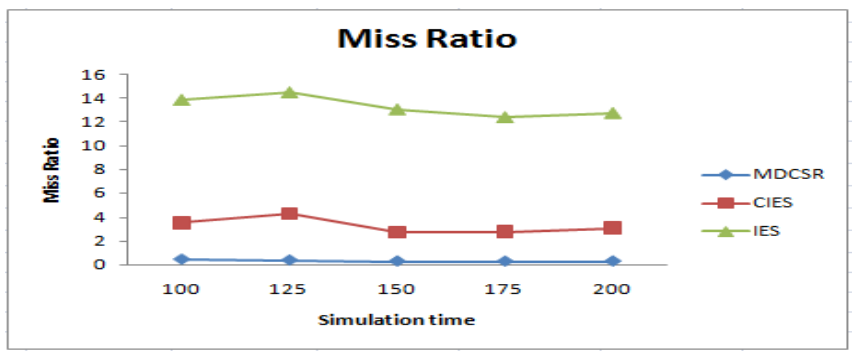

Fig.23. Simulation Time Versus Miss Ratio

Figure 23 depicts that the miss ratio using MDCSR decreases with an increase in simulation time. Since IES performs scheduling at the node level, the miss ratio is comparatively high than CIES and MDCSR. CIES tracks the past error rate at the network level and switches the node to the sleep state, resulting in a small miss ratio value. The MDCSR perform error estimation at DCC in each cluster while CM is concerned with only sensing task and results in the miss ratio's minimal value. 


\section{Conclusion}

For the energy conservation of sensor nodes, there is a need to devise a routing technique considering the clusterbased approach. The proposed MDCSR employ multi-level structure, forming a cluster of nodes at level 1 used for just sensing task. The next level 2 is consisting of $\mathrm{CH}$ nodes that are responsible for data transmission from $\mathrm{CM}$ nodes to PCH nodes at level 2. The use of a cluster-based approach helps to schedule CM nodes using multi DCC based on sensing error. The application of multi-DCC achieves increased throughput with energy conservation and minimal control overhead compared to single DCC as in CIES. Since DCC works at the cluster level has a minimal error rate, miss ratio, and better performance than IES and CIES. Scheduling of node support energy-efficient routing for timecritical applications but demand reliable transmission of data. Also, the parallel transmission of observation errors from each node to the DCC controller is subjected to interference. The minimization of control packet interference to avoid packet drop can be considered as one of the future works. The packet drop due to the dedicated path with MDCSR can be improved using multi-path routing as a future work.

\section{References}

[1] Younis, O., \& Fahmy, S. (2004, March). Distributed clustering in ad-hoc sensor networks: A hybrid, energy-efficient approach. In IEEE INFOCOM 2004 (Vol. 1). IEEE.

[2] Agarkhed, J., Patil Y. D., \& Patil, S. (2020). A Study of Wireless Sensor Networks to Comprehend their Relevance to Different Applications. Journal of Telecommunications and Information Technology,(vol.3)(pp. 1-11)

[3] Patil Y. D., \& Agarkhed, J. (2015). A review on various issues and applications in wireless sensor networks. International Journal of Science and Research, 4(11), 2518-22.

[4] Mainwaring, A., Culler, D., Polastre, J., Szewczyk, R., \& Anderson, J. (2002, September). Wireless sensor networks for habitat monitoring. In Proceedings of the 1st ACM international workshop on Wireless sensor networks and applications (pp. 88-97).

[5] Kumar, P., Arunachalam, V. P., \& Karthik, S. (2012). A Cluster Based Multipath Routing Protocol for Energy Conservation in Wireless Sensor Networks. European Journal of Scientific Research, 78(4), 559-569.

[6] Amrinder Singh and Sandeep Kautish. Study and comparative analysis of various energy efficiency techniques in wireless sensor networks.

[7] Li, Y., Ye, J., \& Zhu, Y. H. (2012). An Energy-Aware Data Gathering Protocol for Wireless Sensor Networks Using Data Correlations. In 2011 International Conference in Electrics, Communication and Automatic Control Proceedings (pp. 75-83). Springer, New York, NY.

[8] Huang, J., Hong, Y., Zhao, Z., \& Yuan, Y. (2017). An energy-efficient multi-hop routing protocol based on grid clustering for wireless sensor networks. Cluster Computing, 20(4), 3071-3083.

[9] Kumar, S., Ranjan, P., Ramaswami, R., \& Tripathy, M. R. (2017). Resource efficient clustering and next hop knowledge based routing in multiple heterogeneous wireless sensor networks. International Journal of Grid and High Performance Computing (IJGHPC), 9(2), 1-20.

[10] Haseeb, K., Bakar, K. A., Abdullah, A. H., \& Darwish, T. (2017). Adaptive energy aware cluster-based routing protocol for wireless sensor networks. Wireless Networks, 23(6), 1953-1966.

[11] Wang, J., Cao, J., Ji, S., \& Park, J. H. (2017). Energy-efficient cluster-based dynamic routes adjustment approach for wireless sensor networks with mobile sinks. The Journal of Supercomputing, 73(7), 3277-3290.

[12] Agrakhed, J., Biradar, G. S., \& Mytri, V. D. (2012, September). Adaptive multi constraint multipath routing protocol in wireless multimedia sensor network. In 2012 International Conference on Computing Sciences (pp. 326-331). IEEE.

[13] Elhoseny, M., Elleithy, K., Elminir, H., Yuan, X., \& Riad, A. (2015). Dynamic clustering of heterogeneous wireless sensor networks using a genetic algorithm, towards balancing energy exhaustion. International Journal of Scientific \& Engineering Research, 6(8), 1243-1252.

[14] Vijayalakshmi, K., \& Anandan, P. (2019). A multi objective Tabu particle swarm optimization for effective cluster head selection in WSN. Cluster computing, 22(5), 12275-12282.

[15] Raman, C. J., \& James, V. (2019). FCC: Fast congestion control scheme for wireless sensor networks using hybrid optimal routing algorithm. Cluster Computing, 22(5), 12701-12711.

[16] He, T., Krishnamurthy, S., Luo, L., Yan, T., Gu, L., Stoleru, R., ... \& Abdelzaher, T. F. (2006). Vigilnet: An integrated sensor network system for energy-efficient surveillance. ACM Transactions on Sensor Networks (TOSN), 2(1), 1-38.

[17] Liu, H., Chandra, A., \& Srivastava, J. (2006, April). eSENSE: energy efficient stochastic sensing framework for wireless sensor platforms. In 20065 th International Conference on Information Processing in Sensor Networks (pp. 235-242). IEEE.

[18] Patil Yogita Dattatraya'a, Jayashree Agarkhed, and Siddarama R Patil.(2015) Error prediction scheduling for energy efficient routing in wireless sensor network. 8(5), 1893-1902.

[19] Zhang, Q., Fu, L., Gu, Y. J., Gu, L., Cao, Q., Chen, J., \& He, T. (2013). Collaborative scheduling in dynamic environments using error inference. IEEE transactions on parallel and distributed systems, 25(3), 591-601.

[20] Luo, H., Wang, J., Sun, Y., Ma, H., \& Li, X. Y. (2010, June). Adaptive sampling and diversity reception in multi-hop wireless audio sensor networks. In 2010 IEEE 30th International Conference on Distributed Computing Systems (pp. 378-387). IEEE.

[21] Yun, Z., Bai, X., Xuan, D., Lai, T. H., \& Jia, W. (2010). Optimal Deployment Patterns for Full Coverage and \$ k \$Connectivity \$(klleq 6) \$ Wireless Sensor Networks. IEEE/ACM transactions on networking, 18(3), 934-947.

[22] Wang, X., Xing, G., Zhang, Y., Lu, C., Pless, R., \& Gill, C. (2003, November). Integrated coverage and connectivity configuration in wireless sensor networks. In Proceedings of the 1st international conference on Embedded networked sensor systems (pp. 28-39). 
[23] Zhou, Z., Das, S., \& Gupta, H. (2004, October). Connected k-coverage problem in sensor networks. In Proceedings. 13th International Conference on Computer Communications and Networks (IEEE Cat. No. 04EX969) (pp. 373-378). IEEE.

[24] Zhang, Q., Gu, Y., He, T., \& Sobelman, G. E. (2008, April). Cscan: A correlation-based scheduling algorithm for wireless sensor networks. In 2008 IEEE International Conference on Networking, Sensing and Control (pp. 1025-1030). IEEE.

[25] Muhammad Noman Riaz, (2018)" Clustering Algorithms of Wireless Sensor Networks: A Survey", International Journal of Wireless and Microwave Technologies(IJWMT), Vol.8, No.4, (pp. 40-53),.DOI: 10.5815/ijwmt.2018.04.03

[26] Saidu, M., Onwuka, E. N., Okwori, M., \& Umar, A. (2016). An enhanced leach routing algorithm for energy conservation in a wireless sensor network. International Journal of Wireless and Microwave Technologies, 6, (pp. 59-71).

[27] Anand Khandare, Abrar Alvi, (2018) "Efficient Clustering Algorithm with Enhanced Cohesive Quality Clusters", International Journal of Intelligent Systems and Applications(IJISA), Vol.10, No.7, (pp.48-57).

[28] Sundani, H., Li, H., Devabhaktuni, V., Alam, M., \& Bhattacharya, P. (2011). Wireless sensor network simulators a survey and comparisons. International Journal of Computer Networks, 2(5), 249-265.

[29] Dattatraya, P. Y., \& Agarkhed, J. (2016, March). Simulation an art of performance evaluation in wireless sensor networks. In 2016 International Conference on Circuit, Power and Computing Technologies (ICCPCT) (pp. 1-5). IEEE.

[30] Agarkhed, J., Dattatraya, P. Y., \& Patil, S. R. (2017). Performance evaluation of QoS-aware routing protocols in wireless sensor networks. In Proceedings of the First International Conference on Computational Intelligence and Informatics (pp. 559569). Springer, Singapore.

\section{Authors' Profiles}

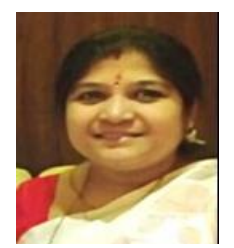

Dr. Jayashree Agarkhed is currently working as Professor in the CSE Department at PDACE Kalaburagi, affiliated with VTU. She obtained her M. Tech in CSE in 2003 and Ph.D. in 2013 from VTU. She has published more than 170 scientific articles in top-tier journals and conferences, including Springer journal, IEEE conference proceedings, and Springer Book chapters. She is the author of 2 Textbooks in the field of computer science from international publishers. She has 'Chaired and Reviewed' various National and International Conferences and is the Reviewer for ELSEVIER, IEEE ACCESS, IEEE Electronics Letters, IET Software, IEEE Transactions on Industrial Informatics, IGI Global. She is an Executive council member of IETE for 2016-18 and a senior member of IEEE. She is the ISTE National Award winner 2019 for the title "Rajarambapu Patil National award for promising Engineering Teacher for Creative Work done in Technical Education". She has received "Award for Research Publication (ARP)", Vision Group of Science and Technology, Department of IT, BT and S\&T, Government of Karnataka, 2018-19. Her main research areas are in Wireless sensor network, multimedia information networks, artificial intelligence, cloud computing, and the Internet of things.

E-mail: jayashreeptl@yahoo.com

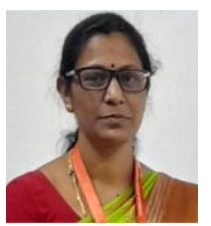

Dr. Patil Yogita Dattatraya is currently working as an Associate Professor, Computer Science and Engineering department, Keshav Memorial Institute of technology, Hyderabad, Telangana. Formerly worked as Professor and Dean of the Faculty of Computer Application at Sharnbasva University, Kalaburagi, Karnataka, India. She has completed her Ph.D., from Poojya Doddappa Appa (PDA) College of Engineering, Kalaburagi, affiliated to Visvesvaraya Technological University (VTU), Belagavi in 2020. She has completed B.E., from the Pune University in 2000 and an M.Tech., in Computer Science and Engineering in 2010 from VTU. Her research area of interest includes Wireless Sensor Network, the Internet of things, and Cloud Computing.

E-mail: agyogita@gmail.com

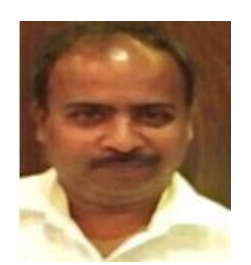

Dr. Siddarama R. Patil received BE degree in Electronics and Communication Engineering from Gulbarga University, Kalburagi, Karnataka, India, M.Tech. in Telecommunication Engineering, and Ph. D from Indian Institute of Technology (IIT), Kharagpur, India in 1990, 1999, and 2009 respectively. Currently, he is a Professor in the Electronics, Communication, and Engineering Department and Dean Academics of Poojya Doddapa Appa College of Engineering, Kalburagi, Karnataka, India. He has published more than 150 research papers in various national and international journals and conferences. He is a life member of the Indian Society for Technical Education (ISTE), India Member of Institute of Electrical and Electronics Engineers (IEEE), Member of Institute of Electronics and Telecommunication Engineers (IETE), India, and Member of Institute of Engineers, India. His current research includes Information Theory and Coding, Turbo Codes, LDPC codes, Iterative decoding algorithms, Wireless Sensor Network, Mobile Ad Hoc Network, and Cognitive Radio.

E-mail: pdapatil@gmail.com

How to cite this paper: Patil Yogita Dattatraya, Jayashree Agarkhed, Siddarama Patil, "Multi Duty Cycle Scheduled Routing in Wireless Sensor Network-lifetime Maximization", International Journal of Computer Network and Information Security(IJCNIS), Vol.13, No.5, pp.55-67, 2021. DOI: 10.5815/ijcnis.2021.05.05 